\title{
PRESTACIÓN DEL SERVICIO DE AGUA POTABLE A TRAVÉS DE COMUNIDADES RURALES, MUNICIPIO DE OCAMONTE. IMPLICACIONES SOCIOJURÍDICAS*
}

\author{
ROBERTO SILVA FERNÁNDEZ** \\ GONZALO ROMERO BECERRA*** \\ José VALENTín PEREIRA RUEDA****
}

Presentado: 27 de octubre de 2013 • Aprobado: 9 de diciembre de 2013

\section{Resumen}

Esta investigación está encaminada hacia el conocimiento de la problemática del servicio de agua potable en las zonas rurales del muni-

\footnotetext{
El artículo es resultado de una investigación terminada que lleva por título "Implicaciones legales de la prestación del servicio de agua potable en los sectores rurales del municipio de Ocamonte, Santander: frente a las posibilidades de su prestación por parte de las comunidades rurales organizadas". El proyecto fue desarrollado por el Grupo de Estudios Constitucionales (GECO) de la Facultad de Ciencias Jurídicas y Políticas, en la línea de investigación: Análisis jurídico y sociojurídico en Derecho Administrativo. Investigador principal: Roberto Silva Fernández. Coinvestigadores: José Valentín Pereira Rueda y Gonzalo Romero Becerra. Investigación financiada por la Fundación Universitaria de San Gil, unisangil. Terminada en el mes de septiembre de 2013.

** Abogado de la Universidad Industrial de Santander; especialista en Derecho Administrativo de la Universidad Libre, sede Socorro; especialista en Docencia Universitaria de la Universidad Nueva Granada; docente en el área de Derecho Público en unisangil; coordinador de investigaciones de la Facultad de Ciencias Jurídicas y Políticas de unisangil; director del Grupo de Estudios Constitucionales, GECO.rsilva@unisangil.edu.co.

*** Estudiante de noveno semestre de Derecho. Integrante del semillero Aguanentinos. Grupo de Estudios Constitucionales, GECO. gonzaloromero91@yahoo.com.co.

***** Estudiante de noveno semestre de Derecho. Integrante del semillero Aguanentinos. Grupo de Estudios Constitucionales, GECO. aguanentinos2011@unisangil.edu.co.
} 
cipio de Ocamonte, Santander. Se centra en aspectos fundamentales, como la cobertura, la calidad del líquido y el estado de la prestación del servicio por parte del ente territorial. Caracteriza la situación problemática e indaga por la posibilidad sociojurídica de que las comunidades rurales organizadas, propietarias de los acueductos tradicionales, puedan prestar el servicio en nombre del Estado. Se efectuó el análisis de la normativa que regula la materia, de los antecedentes y, adicionalmente, se aplicaron instrumentos de exploración para conocer la situación real de los acueductos rurales que abastecen de agua para el consumo humano y el desarrollo de labores agropecuarias, así como la percepción y las perspectivas de los pobladores frente al problema. Como resultado se evidenciaron el abandono estatal en la prestación del vital servicio y la ausencia de políticas e inversiones para subsanar la problemática. También se evidenció la posibilidad jurídica de desarrollar mecanismos que le permitirían a la comunidad rural organizada prestar el servicio de acueducto constituyéndose como asociaciones o empresas comunitarias sin ánimo de lucro y cumpliendo requisitos que la ley les exige a los prestadores.

Palabras clave: agua potable, acueducto rural, servicio público, comunidad organizada, empresa comunitaria.

\section{PROVISION OF WATER THROUGH \\ THE RURAL COMMUNITIES IN THE \\ MUNICIPALITY OF OCAMONTE, SANTANDER. SOCIO LEGAL IMPLICATIONS}

\section{Abstract}

Research aimed to understanding the problems of drinking water in rural areas of the municipality of Ocamonte, Santander, in key areas such as coverage, quality and condition of the fluid delivery by the local authority. Characterizes the problematic situation and explores the legal partner of organized rural communities, traditional owners of the aqueducts, can provide for the State service possible. Was done the analysis of the rules governing the matter, background and further 
exploration tools were applied to understand the real situation of rural aqueducts that supply water for human consumption and agricultural development work, as well as perceptions and perspectives are made of the people with the problem. As a result of government neglect in providing the vital service and lack of policies and investments to address the problem was evident. Was evidenced the legal possibility to develop mechanisms that will allow the rural community to provide water service constituting their self as nonprofit associations or firms and fulfilling requirements that law demand to providers.

Keywords: potable water, rural water supply, public service, organized community, community enterprise.

\section{DISPOSITION DE L'EAU PAR LES \\ COMMUNAUTÉS RURALES, MUNICIPALITÉ DE SANTANDER OCAMONTE: CONSÉQUENCES JURIDIQUES SOCIO}

\section{Résumé}

Recherches visant à comprendre les problèmes de l'eau potable dans les zones rurales de la municipalité Ocamonte, Santander, dans des domaines clés tels que la couverture, la qualité et l'état de la distribution de fluide par l'autorité locale. Caractérise la situation problématique et explore le partenaire juridique des communautés rurales organisées, propriétaires traditionnels des aqueducs, peuvent fournir pour le service possible de l'État. L'analyse des règles régissant la matière, fond et de nouveaux outils d'exploration ont été appliqués à comprendre la situation réelle des aqueducs ruraux qui fournissent de l'eau pour la consommation humaine et de développement agricole, ainsi que les perceptions et les points de vue sont faites des personnes ayant le problème. À la suite de la négligence du gouvernement à fournir le service essentiel et le manque de politiques et d'investissements pour s'attaquer au problème était évidente. La possibilité juridique d'élaborer des mécanismes qui permettent à la communauté rurale organisée fournir un service de l'eau constitué d'associations 
ou d'entreprises et des exigences de conformité de la loi à but non lucratif qui exigent des fournisseurs était évident.

Mots-clés: eau potable, l'approvisionnement en eau en milieu rural, service public, la communauté organisée, l'entreprise communautaire.

\section{FORNITURA DEL SERVIZIO DI ACQUA POTABILE ATTRAVERSO LE COMUNITÀ RURALI, NEL COMUNE DI OCAMONTE SANTANDER: IMPLICAZIONI SOCIO GIURIDICHE}

\section{Riassunto}

La ricerca condotta per conoscere il problema dell'acqua potabile nelle zone rurali del comune di Ocamonte, Santander, in settori fondamentali come la copertura, la qualità del liquido e lo stato di fornitura da parte dell'ente territoriale. Ha caratterizzato la situazione problematica ed indaga la possibilità socio giuridica di che le comunità rurali organizzate, proprietari degli acquedotti tradizionali, possano fornire in nome dello stato il servizio. È stato effettuato l'analisi delle norme che disciplinano la materia, gli antecedenti ed inoltre sono stati applicati gli strumenti di scansione per capire la reale situazione di acquedotti rurali che forniscono acqua per il consumo umano e il lavoro di sviluppo agricola, così come le percezioni e le prospettive delle abitanti rispetto al problema. Di conseguenza ha mostrato l'abbandono dello stato nel fornire il servizio vitale e l'assenza di politiche e investimenti per risolvere il problema. É evidenziato la possibilità legale di sviluppare meccanismi che permettono alla comunità rurale organizzata fornire il servizio idrico costituendosi da associazioni o imprese comunitarie senza scopo di lucro adempiendo i requisiti di conformità alla normativa che richiedono agli fornitori

Parole chiave: acqua potabile; acquedotto rurale; servizio pubblico; comunità organizzata; Impresa comunitaria. 


\section{PRESTAÇÃO DO SERVIÇO DE ÁGUA POTÁVEL A TRAVÉS DAS COMUNIDADES RURAIS, NO MUNICÍPIO DE OCAMONTE SANTANDER: IMPLICAÇÕES SÓCIO-JURÍDICAS}

\section{Resumo}

Esta pesquisa visa conhecer a problemática do serviço de água potável nas zonas rurais do município de Ocamonte, Santander, em aspectos fundamentais como a cobertura, qualidade do líquido e estado da prestação da parte do ente territorial. Caracteriza a situação problemática e indaga a possibilidade sócio-jurídica de que as comunidades rurais organizadas, proprietárias dos aquedutos tradicionais, possam prestar o serviço em nome do Estado. Foi feita a análise da normativa que regulamenta a matéria, os antecedentes e, adicionalmente, foram aplicados instrumentos de exploração para conhecer a situação real dos aquedutos rurais que fornecem a água para o consumo humano e desenvolvimento de trabalhos agropecuários, tanto como a percepção e perspectivas da população a respeito do assunto. Como resultado, foi evidenciado o abandono do estado na prestação do serviço vital e a ausência de políticas e investimentos para corrigir a problemática. Foi evidenciada a possibilidade jurídica para desenvolver mecanismos que permitam à comunidade rural organizada prestar o serviço de água, ao se constituírem em associações ou empresas comunitárias sem fins lucrativos e cumprindo requerimentos que a lei exige aos fornecedores.

Palavras chave: água potável; aqueduto rural; serviço público; comunidade organizada; empresa comunitária. 


\section{INTRODUCCIÓN}

El agua, considerada como un elemento natural, es tan importante y necesaria en el planeta que sin ella los seres vivos no subsistirían. Ello explica las razones que llevaron a diversas civilizaciones a asentarse en lugares próximos a las fuentes de agua o donde las condiciones geográficas les permitieran disponer del recurso. Actualmente las comunidades obtienen el agua mediante sistemas de acueducto, suministrado como servicio público domiciliario.

La prestación del servicio de agua potable hace parte de los cometidos y fines del Estado ${ }^{1}$ contemplados en la Constitución Política de Colombia. En la Carta además se establece como deber del Estado la prestación de los servicios públicos domiciliarios ${ }^{2}$, entre ellos el agua potable y el saneamiento básico. En cumplimiento del mandato, el legislador estableció como competencia de los municipios "asegurar que se presten a sus habitantes, de manera eficiente, los servicios domiciliarios de acueducto, alcantarillado, aseo (...), por empresas de servicios públicos de carácter oficial, privado o mixto, o directamente por la administración central del respectivo municipio" (Congreso de la República de Colombia, 1994, Ley 142). En las cabeceras municipales se presta el servicio de acueducto con un índice de calidad aceptable, situación que contrasta con la prestación en el sector rural, donde en la práctica no existe cobertura debido a que la asignación de recursos es más que precaria, nula.

Frente al carácter que ostenta el agua en relación con los derechos fundamentales, la Corte Constitucional, en sonada sentencia de tutela (1995), establece que:

En principio, el agua constituye fuente de vida y la falta del servicio atenta directamente con el derecho fundamental a la vida de las personas. Así pues, el servicio público domiciliario de acueducto y

\footnotetext{
1 ARTÍCULO $2^{\circ}$. Son fines esenciales del Estado: servir a la comunidad, promover la prosperidad general y garantizar la efectividad de los principios, derechos y deberes consagrados en la Constitución; facilitar la participación de todos en las decisiones que los afectan y en la vida económica, política, administrativa y cultural de la Nación; defender la independencia nacional, mantener la integridad territorial y asegurar la convivencia pacífica y la vigencia de un orden justo.

2 ARTíCULO 365. Los servicios públicos son inherentes a la finalidad social del Estado. Es deber del Estado asegurar su prestación eficiente a todos los habitantes del territorio nacional.
} 
alcantarillado en tanto que afecte la vida de las personas, la salubridad pública o la salud, es un derecho constitucional fundamental.

(Sentencia T. 413 de 1995)

Pese a ser un cometido del Estado la prestación del servicio de agua potable con niveles de óptima calidad a todos los habitantes del territorio, lo cierto es que el vital recurso no se ha llevado a las áreas rurales a través de las empresas prestadoras de servicios públicos. No obstante, en todo el país las comunidades rurales se lo proveen de manera artesanal, acudiendo al aprovechamiento de fuentes hídricas naturales, tales como aljibes, arroyos y pozos. Con incipientes infraestructuras se han organizado como comunidades alrededor de acueductos comunitarios que acopian el recurso captado de las fuentes ya mencionadas y conducen el agua, a través de una manguera convencional o empleando otros métodos rudimentarios, hasta los predios de los usuarios.

Llegando al espacio geográfico objeto del estudio se observó que las comunidades rurales del municipio de Ocamonte, Santander, ante el abandono estatal, se han organizado y construyeron sus propios sistemas de acueducto. El servicio por autogestión resulta relativamente económico (aunque sin las condiciones organolépticas que requiere el agua para el consumo humano) y de suma utilidad para las actividades agropecuarias que requieren del preciado líquido con aceptable nivel de cobertura para los asociados. Lo cierto es que, aunque de manera precaria, la propia comunidad se autoprovee el servicio. Sin embargo, las condiciones del líquido en términos organolépticos no cumplen con las mínimas condiciones, y suministrarlo con los parámetros exigidos resulta para las comunidades en extremo oneroso e imposible de realizar, si se tienen en cuenta la infraestructura requerida y las distancias entre los usuarios. Pero las entidades territoriales tampoco han asumido la responsabilidad de este suministro, pese a la obligación constitucional, por causas similares, aunque sí han elucubrado sobre la posibilidad de que sean las empresas particulares las que presten el servicio, lo cual es también irrealizable por las dificultades anotadas.

Con todo, y como complemento a lo que se ha establecido anteriormente, los esfuerzos de las comunidades con sus acueductos (que en algo subsanan la ausencia del Estado) se ven amenazados, y la autonomía que tienen sobre el sistema de autoprestación podría llegar a su fin, merced a la perentoria exigencia legal a los municipios para que asuman la prestación del servicio (Ley 142 de 1994), puesto que tal cambio implica la dirección 
y el manejo de los sistemas por parte del ente territorial (Decreto 398 de 2002). Lo cierto es que para los municipios resulta más conveniente no asumir la prestación del servicio, debido a que no cuentan con los recursos ni la capacidad para construir la infraestructura requerida para acopiar el agua en un solo lugar y luego conducirla a cada hogar campesino o construir un sinnúmero de subsistemas de potabilización para llevar el preciado líquido a toda el área rural.

Frente a la situación problemática expuesta, revisando los antecedentes, se evidenció la ausencia de avances sobre el tema en concreto. En tal sentido, resultaba necesario y plausible adelantar la investigación por razones de naturaleza social, económica y jurídica. Partiendo de lo expuesto se abordó el ejercicio investigativo y se trazó el objetivo general de establecer las implicaciones sociojurídicas que conlleva la prestación del servicio de agua potable a través de las comunidades rurales organizadas como empresas sin ánimo de lucro en el municipio de Ocamonte, Santander. El cometido se abordó desde varios aspectos, a saber: a) La exigencia legal a las entidades territoriales para que asuman la prestación de servicios públicos domiciliarios en las zonas rurales. b) La existencia de sectores rurales que cuentan con sistemas propios de acueducto, pero sin asistencia estatal. c) El vacío normativo que no le permite al Estado destinar recursos para inversión en la asistencia y subsidio del servicio prestado por las propias comunidades. d) El desconocimiento de la regulación normativa y de las exigencias organolépticas y técnicas. e) La dificultad que supondría la potabilización de la totalidad del agua, con el consiguiente perjuicio para ciertas labores agropecuarias.

La investigación encuentra justificación en la necesidad de hallar un modelo jurídico de organización o empresa comunitaria, que pueda acceder a los recursos públicos. Resulta incluso de incontestable beneficio para el legislador que se halle la solución más justa y eficaz, y también para la academia, como generador de conocimiento útil para el progreso social de las comunidades.

Se trata de una investigación de carácter sociojurídico en cuanto indaga en los espacios vitales de las comunidades acerca de la prestación del servicio público de agua como problema social, en busca de una solución jurídica. Lo anterior implica que la investigación sea también descriptiva y analítica. Por su propósito es también de corte cualitativo, puesto que lo que 
se busca es examinar y explicar el fenómeno social según los tópicos legal y fáctico.

El ejercicio investigativo encontró en el ordenamiento jurídico viabilidad para aplicar diversos modelos de constitución, con el fin de que la comunidad organizada pudiera prestar el servicio de suministro de agua (Decreto 421 de 2000). Además se pudo conocer la situación real de la prestación del servicio y se observó que la comunidad está organizada a nivel veredal en pequeñas asociaciones relativamente legalizadas, pero que no gozan de la asistencia presupuestal por parte del municipio.

La investigación comporta un carácter sociojurídico en cuanto reviste una problemática actual de abordaje multifactorial e interdisciplinar. Teniendo en cuenta el problema social, se traduce en una investigación descriptiva, con matices de investigación teórica aplicada. La investigación es también de tipo cualitativo, en cuanto busca examinar la naturaleza del fenómeno de tipo legal y práctico en la prestación del servicio de agua potable a la población objeto de estudio. Involucra la recolección de datos, la aplicación de técnicas de revisión documental, encuestas, entrevistas y análisis, con el propósito de convertir esta información en categorías de análisis que permitan obtener una apreciación objetiva con respecto al estado y la calidad en la prestación del servicio, para llegar a diseñar posibles fórmulas de solución para la situación problemática. El estudio contó con una recolección de información proveniente de diversas fuentes, entre ellas el Esquema de Ordenamiento Territorial, el Plan de Desarrollo Municipal y encuestas a la comunidad en busca de información primaria acerca de la realidad social en torno al servicio, la cantidad de acueductos, de usuarios, las empresas constituidas, la calidad del agua y demás aspectos relevantes.

Siguiendo con el diseño de la investigación, en la primera fase (que inició en el primer semestre de 2012) se realizó la recopilación y el análisis de la normatividad que regula la materia, luego un rastreo de los antecedentes y la evolución de los modelos que la norma contempla para el manejo de los servicios públicos domiciliarios; se examinó en general su aplicación en los diferentes escenarios espacio-temporales de la región involucrada en la problemática, hasta llegar al municipio de Ocamonte, escogido como universo de la muestra. Allí se indagó a los representantes de las comunidades veredales, en cuanto conocedores de la información pertinente para el estudio. En reuniones realizadas con los líderes y representantes de la comuni- 
dad rural, a quienes previamente se les encomendó recabar datos precisos de sus comunidades, se recogió la situación actual del suministro de agua. En otro momento o estadio de la investigación se emplearon instrumentos tipo entrevista con funcionarios tanto del municipio como del Ministerio Público, el Concejo y la Corporación Autónoma Regional de Santander. Con los datos recolectados en cada etapa del proceso se realizó un análisis triangular sistemático y holístico, que arrojó certeros elementos para una adecuada comprensión y una adecuada explicación del objeto de investigación, así como un modelo jurídico de solución.

\section{LA SITUACIÓN ACTUAL EN LA INFORMACIÓN OFICIAL}

El municipio de Ocamonte cuenta con una población rural de 3.815 habitantes distribuidos en 21 veredas, frente a 634 habitantes en la zona urbana. Respecto al sector de agua potable y saneamiento básico, el Plan de Desarrollo del Municipio de Ocamonte $(2012$ - 2015) presenta información acerca de la situación actual en la prestación del servicio de acueducto en todo el municipio, destacando que el número de viviendas en el sector urbano es de 194 y en el sector Rural 1063 para un total de 1257 viviendas (Ocamonte, 2012, p. 57). Respecto a la cobertura del servicio de acueducto, en el sector urbano 3 no tienen, en tanto en el sector rural 231 carecen del servicio; el porcentaje de viviendas con conexión domiciliaria de acueducto en la zona urbana (prestado por el municipio) es del $98 \%$. Y en la zona rural es del $78.26 \%$ (Por autosuministro); el 21.72\% de las viviendas equivalente a 231, no cuenta con el servicio (Ocamonte, 2012, p. 58).. Claramente se observa una desproporción respecto a la cobertura y la asistencia estatal en los dos sectores, lo cual evidencia la situación problemática que planteó el proyecto de investigación.

El porcentaje de viviendas con conexión domiciliaria de acueducto en la zona urbana (prestado por el municipio) es del $98 \%$. Y en la zona rural es del $78.26 \%$ (por autosuministro); el 21.72\% de las viviendas, equivalente a 231, no cuenta con el servicio. Claramente se observa una desproporción respecto a la cobertura y la asistencia estatal en los dos sectores, lo cual evidencia la situación problemática que planteó el proyecto de investigación.

El municipio de Ocamonte cuenta con una enorme riqueza en fuentes hídricas, que el Plan de Desarrollo 2012-2015 relaciona. Y dada su importancia para esta investigación se considera relevante describirla: 
La red hidrográfica municipal se localiza sobre la margen derecha del río Fonce y está constituida, en orden de importancia, por el río Taquiza, las quebradas Moraría, El Salitre, Agua Fría, El Tunal, El Fique y drenajes que conforman la parte alta de la quebrada Sobacuta, en el extremo norte del municipio. Se destaca la microcuenca de la quebrada Moraría, que abarca un $45 \%$ del área municipal y conduce sus aguas al río Fonce, atravesando el municipio desde su extremo nororiental hacia el suroccidente después de recoger aguas abajo, desde su nacimiento por la margen izquierda, las quebradas La Honda, Bejuco, San Bernardo, Garabito, La Lejía, El Pinal, Agua Blanca, Las Piedras y Las Picas. De todas ellas las más importantes son Las piedras y La Lejía. Por la margen derecha aguas abajo recoge las aguas de las quebradas Las Flores, La Laja, El Hatigal, El Cajón, Los Iraquíes, La Honda y Germania. Como se aprecia, la riqueza de fuentes de agua hace de Ocamonte un municipio de alto potencial hídrico en la región, razón por la cual, y dada la pluricitada importancia del agua, se debería ofrecer un manejo integral para su conservación.

El plan para el cuatrienio 2012-2015 proyecta la construcción y la reparación del sistema de acueducto, construyendo un acueducto para aumentar la cobertura del servicio. Así mismo plantea el desarrollo de un programa de rehabilitación de sistemas de potabilización del agua que busca reducir el $16 \%$ del IRCA ${ }^{3}$ en el acueducto urbano, y menciona el proyecto de adecuar tres plantas de tratamiento de agua potable, implementar un programa de macro y micromedidores, construir tanques de almacenamiento e implementar un programa para el uso eficiente y el ahorro del agua. Lo cierto (se anota con preocupación) es que los planes del gobierno local no contemplan al sector rural como destinatario del servicio de agua potable.

\section{LA INFORMACIÓN REAL RESULTANTE DE LAS ENCUESTAS}

Como técnica de recolección de información se aplicó una encuesta cuya finalidad era la de conocer la realidad material en la percepción de la comunidad frente a la prestación del servicio de agua ofrecido por la comunidad organizada en el área rural del municipio de Ocamonte y la

3 Índice de riesgo de la calidad del agua para el consumo humano. 
de generar respuestas al problema inicialmente planteado. El instrumento ${ }^{4}$ fue empleado con 23 dirigentes del área rural, que fungen como directivos o coordinadores de organizaciones comunitarias que actualmente operan como prestadoras del servicio de agua en las veredas del municipio de Ocamonte. Los mencionados conocen la situación problemática de toda el área rural objeto de estudio y por tal razón se consideran, para esta investigación, representantes de la población. La principal razón para escoger a este grupo poblacional ha sido el rol que juegan como líderes administradores de los sistemas de acueducto rural y, en esa medida, como conocedores de los aspectos relevantes para la investigación. Por medio de estos líderes y representantes se han obtenido también datos precisos acerca de la situación de los habitantes de todas las veredas, en relación con la problemática del suministro de agua.

En la información oficial contenida en el Plan de desarrollo del municipio de Ocamonte, Santander, 2012-2015, se relaciona un total de 21 veredas pertenecientes a la jurisdicción del municipio de Ocamonte.

\begin{tabular}{|c|c|}
\hline Nro. & VEREDA \\
\hline 1 & Aguablanca \\
\hline 2 & Aguafría \\
\hline 3 & Buenavista \\
\hline 4 & El Rincón \\
\hline 5 & El Río \\
\hline 6 & Guaduas \\
\hline 7 & Hatigal \\
\hline 8 & Hatillo \\
\hline 9 & La Cañada \\
\hline 10 & Las Flores \\
\hline 11 & La Laja \\
\hline
\end{tabular}

\begin{tabular}{|c|c|}
\hline Nro. & VEREDA \\
\hline 12 & Miraflores \\
\hline 13 & Morario \\
\hline 14 & Olvido Monchía \\
\hline 15 & Puentes \\
\hline 16 & Puertas \\
\hline 17 & San Bernardo \\
\hline 18 & San Rafael \\
\hline 19 & El Centro \\
\hline 20 & Copero \\
\hline 21 & El Palenque \\
\hline
\end{tabular}

Fuente: Plan de desarrollo del municipio de Ocamonte 2012-2015

4 Se trata de un cuestionario diseñado por el investigador, fundamentando sus variables e indicadores en el marco de referencia, que se aplicó a una muestra determinada para poblaciones finitas referenciada por Munch (1995), citado por Davis (2001), en donde se estableció una confiabilidad del $90 \%$ y un error del $5 \%$, con una probabilidad a favor de 0,5 y otra en contra de 0,5 . 
La información obtenida desde la comunidad arroja un total de 1245 familias $^{5}$ en el sector rural del municipio de Ocamonte.

\section{CONCENTRACIÓN PORCENTUAL DE POBLACIÓN DE FAMILIAS POR VEREDA}

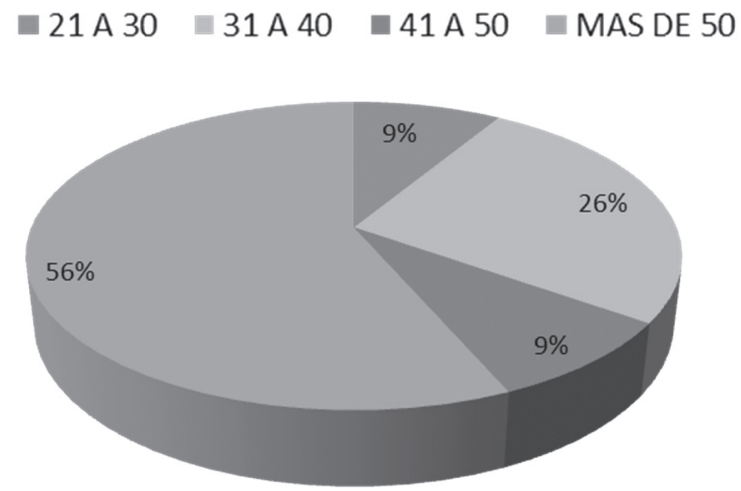

Figura 1. Localización de la población

Fuente: encuesta realizada por los autores

Conforme a la gráfica, la población se encuentra asentada de manera heterogénea, así: el $56 \%$ corresponde a las veredas que cuentan con más de 50 familias; el $26 \%$ corresponde a las veredas que cuentan con una concentración de entre 31 y 40 familias; el $9 \%$ corresponde a las veredas que cuentan con una concentración de entre 21 y 30 familias; el $9 \%$ corresponde a las veredas que cuentan con una concentración de entre 31 y 40 familias.

La información presentada permite evidenciar que la población se encuentra dispersa de manera desigual en cada vereda, lo cual indica que en unas veredas existe mayor demanda del recurso hídrico y, en consecuencia, más obra de infraestructura de conducción.

5 El número de familias puede variar al confrontar esta información con el número de viviendas que registra el plan de desarrollo, y esto se debe a que en algunas viviendas reside más de una familia. 


\section{COBERTURA DE LOS ACUEDUCTOS RURALES COMUNITARIOS}

De 1245 familias, 423 no cuentan con suministro de agua por parte de los acueductos comunales y se proveen de agua acarreándola ya sea de fuentes cercanas o de aljibes propios. En la gráfica se aprecia en términos porcentuales.

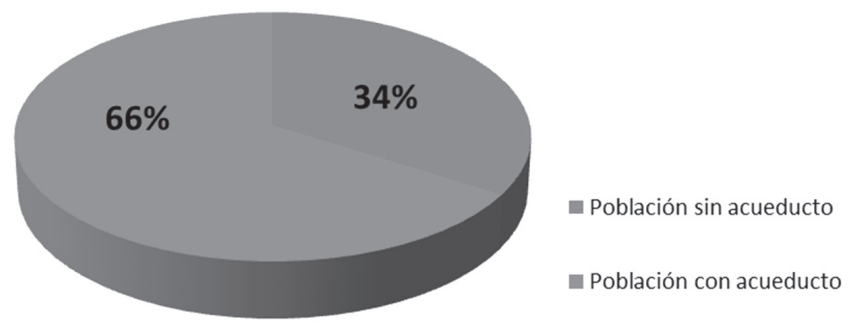

Figura 2. Cobertura de los acueductos rurales comunitarios

Fuente: encuesta realizada por los autores

\section{TIPO DE CAPTACIÓN O FUENTE QUE SURTE LOS ACUEDUCTOS CO- MUNITARIOS}

De la información presentada se deduce que la zona rural del municipio cuenta con abundantes fuentes de aguas superficiales que discurren por los sectores conforme a la topografía, lo cual facilita de alguna manera la captación.

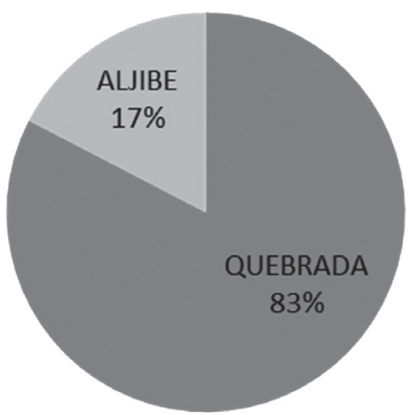

Figura 3. Tipo de captación o fuente que surte los acueductos comunitarios

Fuente: encuesta realizada por los autores 


\section{Material utilizado en las redes de conducción}

Los datos que anteceden son importantes para destacar que en una porción importante de las redes de conducción se usa manguera convencional, lo cual no es permitido por la Superintendencia de Servicios Públicos en el caso de los acueductos, toda vez que se ha considerado como un material altamente contaminante y como tal perjudicial para la salud humana. Lo anterior significa que las comunidades que aún utilizan la manguera convencional deberán realizar una importante inversión con el fin de reemplazar dicho material por uno autorizado que no presente riesgos para la salud.

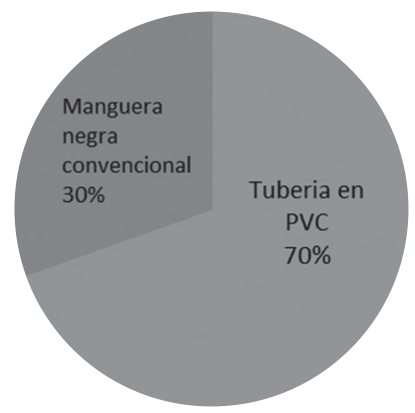

Figura 4. Material utilizado en las redes de conducción

Fuente: encuesta realizada por los autores

\section{Potabilidad del agua que llega a los hogares campesinos}

El resultado es preocupante, ya que el requisito fundamental para que una empresa o la comunidad puedan prestar el servicio de agua en forma autónoma es que esta sea apta, en términos organolépticos, para el consumo humano. En la gráfica se evidencia que el $4 \%$ de los usuarios utilizan plantas de tratamiento, el $35 \%$ lo efectúa con su propio método ${ }^{6}$ y en la mayoría de los acueductos no se aplica ningún sistema de purificación; se evidencia que el agua utilizada se toma directamente de la fuente de captación, lo que

6 Mediante filtros artesanales o hirviendo el líquido. 
genera un riesgo para la salud de la población ${ }^{7}$. Cabe anotar, sin embargo, que el agua usada para riego y demás faenas agrícolas no requiere potabilización y, al contrario, potabilizar el agua para esos usos resultaría nocivo ${ }^{8}$.

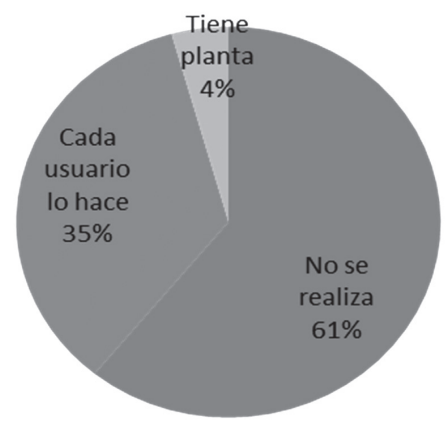

Figura 5. Potabilidad del agua que llega a los hogares campesinos

Fuente: encuesta realizada por los autores

\section{PERCEPCIÓN DE LA COMUNIDAD RURAL SOBRE EL FUTURO DE SUS ACUEDUCTOS}

Se les preguntó a los dirigentes y representantes de la comunidad acerca de si estarían dispuestos a entregarle al municipio la propiedad del acueducto de sus veredas, para que este asuma la prestación del servicio, y las respuestas fueron concluyentes: ningún miembro de estas comunidades le entregaría sus acueductos al municipio u otra entidad. En este punto se observa que desde siempre las comunidades han asumido de manera directa la prestación del servicio. Se evidencia además que lo están haciendo de manera organizada, y que el ejercicio administrativo les permite ver que los beneficia aplicar un modelo solidario donde no exista el ánimo lucrativo,

7 Riesgos que resultan de la contaminación que se genera cuando en los sitios de captación hay vertimiento de desechos debido a procesos como los aplicados al lavado del café, al ganado que abreva en estos sitios y a las actividades de habitantes que procesan café en las fuentes, procesan materiales como el fique o hacen otros usos directos de las fuentes de agua altamente nocivos.

8 Obsérvese por ejemplo el faenado del café, específicamente el despulpe o descerezado y el lavado, para el cual elementos químicos anexos al agua, como el cloro, resultarían arruinando el proceso y el producto. 
además de que este modelo les permite gestionar de manera autónoma soluciones con respecto a la consecución del valioso recurso, así como el control íntegro de todas las actividades, incluso la propiedad de la infraestructura del sistema.

\section{LAS COMUNIDADES ESTARÍAN DISPUESTAS A POTABILIZAR EL AGUA}

Se indagó en la comunidad acerca de si estarían dispuestos a potabilizar el agua de su acueducto conforme a las disposiciones legales y las establecidas por la Superintendencia de Servicios Públicos, y la respuesta fue unánime y contundente: sí. El resultado denota un alto grado de conciencia de las comunidades y la respuesta obedece no solo a que este es un requisito legal, sino a la necesidad de aplicar un sistema de tratamiento que redunde en beneficios para la salud de quienes consumen el agua. Resta, sin embargo, determinar cuál sería el sistema más adecuado, que al tiempo les permita recibir una conducción de agua sin potabilizar, para actividades agropecuarias, y una para el consumo humano.

\section{LA OPINIÓN DE LAS AUTORIDADES Y DE OTROS SERVIDORES PÚBLICOS}

Ha sido en extremo útil sondear la opinión de diferentes funcionarios que se encuentran involucrados en la problemática del abandono de las comunidades rurales por parte del Estado.

La Personería Municipal, a través de su titular, expone que conforme a las directivas expedidas por la Procuraduría General de la Nación se adelantan gestiones con el fin de verificar la posible contaminación del agua. Manifiesta que, con ocasión de adelantar el Plan Maestro de Aguas, el líquido que surte en estos momentos el acueducto de la cabecera municipal presenta cierto grado de contaminación debido a las obras que se están desarrollando. Añade que en el municipio el agua presenta un grado de contaminación acorde a las condiciones ambientales, pero no de tal magnitud que ponga en riesgo o amenace el derecho fundamental que por conexidad reviste el agua. Manifiesta que no se tiene noticia de que las fuentes hídricas presenten focos de contaminación, a pesar de que no se adelantan en el momento campañas que prevengan tal situación. Es enfático en señalar que actualmente en el sector rural la población no goza del servicio de agua potable prestado por el municipio. 
La Secretaría de Planeación Municipal indica que para el sector rural actualmente hay formulados dos proyectos que consisten en la adecuación de tanques de almacenamiento para las veredas de la parte alta y la ampliación de redes de distribución. Considera que los usuarios, una vez entrado en ejecución el proyecto, podrán acceder a mayores volúmenes de agua, pero deberán asumir los costos de la operación. Respecto a la potabilización del agua manifiesta que en el sector rural no se está haciendo nada.

La administración encuentra que la misma comunidad no ve viable que se potabilice el agua en sus veredas debido a que en muchas partes están produciendo café orgánico. Al potabilizar el agua se utilizaría cloro y el café orgánico se dañaría con este producto, lo que les ocasionaría un daño a los productores del grano. Ante la dificultad manifestada se ha pensado en llevar un proyecto de potabilización pero directamente a las familias, que se ejecute en la entrada de sus casas. La administración manifiesta que para adelantar un proyecto masivo de potabilización del agua a través de plantas se tendría que acudir directamente a la comunidad, lo que volvería muy oneroso el servicio por los costos de operación. Además, el agua no fluiría todo el tiempo en las mismas condiciones y necesitaría un tratamiento diferente. Considera importante poder desarrollar un sistema de potabilización familiar, implementando filtros pequeños con características orgánicas con los cuales los usuarios mismos podrían potabilizar el agua que consumen, manteniendo un bajo costo.

A su turno, el Presidente del Concejo Municipal manifiesta que en el interior de la corporación que preside se ha pensado en brindar todo el apoyo necesario a la comunidad que presta el servicio de acueducto, dado que los acueductos son fuente vital para el desarrollo de la misma comunidad. Por tal razón se está formulando un proyecto que propende por el mejoramiento del servicio, con la búsqueda de la reconstrucción de los acueductos actuales en las diferentes veredas y de la ampliación de cobertura.

Por parte de los funcionarios de la Corporación Autónoma Regional, CAS, se conoció que desde hace quince años aproximadamente se han venido adelantando con las comunidades (especialmente con las JAC) los procesos de legalización del uso del agua, al tiempo que se han brindado las pautas a seguir para la conservación de las fuentes. Estos funcionarios manifiestan que a aquellos encargados de acueductos que aún no han entrado en la legalidad se les ha venido concediendo plazos para que presenten la documentación que los acredita como una JAC, ya sea como asociación o como corporación. 
Con respecto a la gestión de la CAS para fomentar el cuidado y la conservación de las fuentes hídricas, indican que una vez la Corporación otorga las concesiones de agua, con los actos administrativos que generan el permiso, se contrae una serie de obligaciones, entre ellas el manejo adecuado de las aguas, el uso racional, el uso sostenible y las obligaciones de conservar y preservar esas fuentes mediante la construcción de cercas protectoras o áreas de reserva, además de adecuar de la mejor manera posible la infraestructura del sistema de acueducto para evitar el desperdicio del agua.

Es claro que las autoridades territoriales y el Concejo Municipal conocen la problemática, y en tal sentido manifiestan la intención o refieren estar pensando en abordar o implementar soluciones que, sin embargo, no contienen el propósito de asumir el cometido estatal de prestar el servicio público de agua en las zonas rurales, tal como está ordenado por la norma constitucional. Es claro, como lo manifiesta el Secretario de Planeación Municipal, que asumir la prestación del servicio en el sector rural sería en extremo oneroso, tanto para la entidad territorial como para los usuarios del servicio. La personería, como representante del Ministerio Público, defensor y garante de la observancia de los derechos fundamentales, es parca en sus opiniones y solo manifiesta que el sector rural no goza del servicio de suministro de agua potable por parte del municipio. La Corporación está más preocupada (y en razón de sus funciones es natural que así sea) por el control sobre las captaciones ilegales y el cumplimiento de los planes de manejo ambiental y de protección de cuencas.

\section{A manera de corolario}

Se ha evidenciado una problemática que consiste en el abandono estatal que padecen los sectores rurales del municipio de Ocamonte (aunque en realidad se trata de un problema nacional), pero también se comprobó que con la implementación de los sistemas de acueductos comunitarios veredales las comunidades organizadas han suplido relativamente en sus regiones la ausencia del Estado en cuanto a la prestación de los servicios públicos domiciliarios, específicamente el relacionado con el suministro de agua, imprescindible para el desarrollo de la vida en condiciones dignas.

La Ley 388 de 1997, conocida como ley de ordenamiento territorial, detalla los elementos a tener en cuenta para el adecuado manejo de los temas esenciales para el desarrollo sostenible y ordenado. Esta ley tiene en cuenta 
el componente rural para el tema de la prestación de servicios básicos, a lo cual se refiere en el artículo 14, numeral 6, que establece que el componente rural deberá contener la determinación de los sistemas de aprovisionamiento de los servicios de agua potable y saneamiento básico de las zonas rurales a corto y mediano plazo y la localización prevista para los equipamientos de salud y educación.

A partir de la lectura de este aparte normativo, brevemente enunciado, se podría aventurar que todos los elementos jurídicos están dados para que el Estado cumpla con los fines que la Constitución le ha encargado. No obstante, la realidad es que los sectores rurales padecen en grado sumo el abandono por parte del Estado (representado en sus entidades territoriales), mientras que en las zonas urbanas existen niveles aceptables de cobertura.

Los habitantes del área rural del municipio de Ocamonte se han organizado alrededor de sus necesidades $y$, de manera precaria e incipiente, abordan la prestación del servicio de acueducto aplicando un modelo de autogestión comunitaria y solidaria mediante la unión de esfuerzos, para satisfacer en lo básico las necesidades de su conglomerado. El Estado, a quien le asiste el deber de prestar los servicios públicos y abordar la debida planificación y disposición de recursos económicos para otorgar cobertura y fortalecer el servicio, brilla por su ausencia.

La prestación del servicio de acueducto, según la naturaleza jurídica del ente que la realice, implica diferencias sustanciales que inciden en la calidad y en los costos. Es decir, cuando la empresa que asume la prestación del servicio es de naturaleza privada, el determinante es el ánimo de lucro. Si se trata de empresas públicas, el cometido entraña el cumplimiento de un deber o un fin estatal, y si se trata de la misma comunidad impulsada por el propósito de satisfacer necesidades esenciales, se constituyen como entidades sin ánimo lucrativo. La Corte Constitucional (C-741-2003) ha sido prolija al referirse a las organizaciones autorizadas para prestar servicios públicos domiciliarios, y ha conceptuado que la actividad de tales organizaciones que participan en la prestación de los servicios públicos debe orientarse hacia el mejoramiento de la calidad de vida de sus asociados y de la comunidad en general, así como hacia el logro de fines altruistas en favor de grupos marginados o discriminados, sin que ello signifique que su objeto no pueda comprender el que la prestación de servicios públicos se lleve a cabo con eficiencia y calidad, en beneficio también de los usuarios de los mismos.

La investigación, al realizar el proceso de exploración normativa, ha podido concluir que el ordenamiento jurídico colombiano establece quiénes 
pueden ser entes prestadores de servicios públicos. La Constitución Política enuncia como una finalidad del Estado la prestación de servicios públicos (artículos 365 a 370) y establece que estos podrán ser prestados por el Estado, por comunidades organizadas o por particulares. A su turno, respecto a los servicios públicos domiciliarios, la Ley 142 de 1994 establece que las personas que prestan servicios públicos pueden ser organizaciones autorizadas conforme a esta ley para prestar servicios públicos en municipios menores, en zonas rurales y en áreas o zonas urbanas específicas.

En este punto se hace necesario aclarar que la expresión "organizaciones autorizadas" no se puede asimilar a la de "comunidades organizadas" de la que trata la Constitución Política. La razón es que la primera alude al género y puede comprender un conjunto de agentes prestadores, dentro de los cuales se ha de incluir a los particulares que se organizan para la prestación del servicio, pero no necesariamente a través de la figura de empresa; también comprende las organizaciones de economía solidaria, si se entiende que la ley no se refirió en forma expresa a las comunidades organizadas. Se interpreta que estas se encuentran comprendidas dentro de la denominación organizaciones autorizadas.

Conforme a lo establecido en la Ley 142 de 1994, artículo 15.4, se faculta a las organizaciones autorizadas para prestar servicios públicos en municipios menores, en zonas rurales y en áreas o zonas urbanas específicas. Esta norma fue reglamentada por el Decreto Nacional 421 de 2000, que en su artículo 1 contempla que:

Para los efectos de lo establecido en la Ley 142 de 1994, en cuanto a los servicios públicos de agua potable y saneamiento básico, podrán prestar dichos servicios en municipios menores, zonas rurales y áreas urbanas específicas, las comunidades organizadas constituidas como personas jurídicas sin ánimo de lucro (Ministerio de Desarrollo Económico, 2000, Decreto Nacional 421).

Dentro de esta modalidad de empresa se encuentran: las juntas de acción comunal, las juntas administradoras, las asociaciones de usuarios, las corporaciones, las fundaciones y todas las asociaciones.

\section{CONCLUSIONES}

El ejercicio no solamente se limitó a determinar puntualmente la especificidad de los objetivos, sino que permitió establecer cuáles son los ele- 
mentos que con juiciosa aplicación pueden contener las respuestas a la situación problemática en estudio, para beneficio de las comunidades rurales en lo relativo a la cobertura y el mejoramiento en la prestación del servicio público de agua potable.

El tema más vulnerable que se evidenció es el relacionado con la calidad del agua, puesto que no se le somete a procesos o tratamientos. Los funcionarios entrevistados dan cuenta de que la misma comunidad es renuente a la idea de potabilizar toda el agua que usa habitualmente, pues argumenta que no se requiere y que, al contrario, resulta perjudicial para los procesos agropecuarios. Sin embargo consideran que una salida viable es la de aplicar un sistema de potabilización solo para el agua de consumo humano, y dejar sin tratar la de uso en faenas agrícolas. Algunas familias utilizan un sistema de purificación del agua mediante filtros que se consiguen en el mercado.

Durante el proceso se evidenció que la gran masa de población del municipio de Ocamonte se concentra en el sector rural. Los ejercicios e instrumentos de observación permitieron verificar que actualmente no hay una cobertura total del servicio de acueducto en el sector rural y que se suministra por los mismos pobladores. El diagnóstico del Plan de Desarrollo Municipal en el momento de su formulación reportó que 231 familias, que corresponden al $22 \%$ (este dato no concuerda con la información recolectada en la presente investigación) del total rural, no cuentan con el servicio (teniendo en cuenta que el suministro no lo presta el municipio), en tanto que en el área urbana hay un cubrimiento del 98\%. Se resalta el desequilibrio respecto a la cobertura en los dos sectores, lo que indica cierto grado de desatención estatal.

Los usuarios acceden al servicio de agua mediante la conexión a sistemas de acueducto incipientes, y la calidad del agua es deficiente ya que se presentan focos de contaminación en las fuentes hídricas.

La indagación realizada con las juntas administradoras de los acueductos de Ocamonte indica que están operando bajo el marco de la legalidad. No obstante, adolecen de ciertos requisitos que están contemplados en la abundante dispersión normativa que regula la materia, verbi gratia, el Registro Único de Prestadores de Servicios Públicos.

Del ejercicio analítico del ordenamiento jurídico que regula la materia de los servicios públicos se concluye que el Estado, a través de los municipios, está obligado a prestar el servicio de agua potable también en las zonas rurales, ya sea directa o indirectamente, sin perjuicio de que se haga a través de la empresa privada. 
El ordenamiento le permite a la comunidad organizada prestar el servicio público de acueducto en áreas rurales siempre que se constituyan como entidades sin ánimo de lucro, con el cumplimiento de requisitos específicos. Es necesario destacar que esta conformidad con la norma resulta importante porque así los recursos del Estado se pueden entregar a los prestadores del servicio para que cumplan su cometido.

Una gran cantidad de familias (34\%) no están conectadas a los sistemas de abastecimiento, y en general se observó que los sistemas de acueductos son precarios y artesanales en su infraestructura; adicionalmente, son construidos con materiales de conducción no aprobados por las normas técnicas.

Las comunidades, como prestadoras del servicio, cuentan con un modesto sistema administrativo, y aparecen formal pero relativamente legalizadas en forma de grupos o asociaciones que se constituyeron como organizaciones comunitarias bajo el imperio de sus propios estatutos, y cuentan con reconocimiento en el registro de la Cámara de Comercio.

Las comunidades no están dispuestas a entregar el control ni el manejo de sus acueductos al ente municipal, y este tampoco está en capacidad de asumir la prestación del servicio. No cursan proyectos a nivel local para resolver o mitigar el problema.

Que las comunidades rurales básicamente estén reemplazando al Estado en el cumplimiento de uno de sus principales fines, como es el de la satisfacción de necesidades mediante la prestación del servicio de agua, implica la asunción de un rol que no se les está reconociendo a los prestadores del servicio, y lo que es más delicado, estos no están recibiendo los recursos que tienen destinación específica para este cometido. Lo anterior solo es subsanable por parte del mismo Estado, si se facilita la legalización de estas asociaciones en los términos de la Ley 142 de 1994, al punto en que sean los destinatarios de los recursos mencionados.

El Ministerio Público es tímido en su intervención a favor de la comunidad rural y la autoridad ambiental cumple una función de vigilancia para facilitar la legalización del uso del agua, además de que ejerce un control preventivo en su uso racional. 


\section{REFERENCIAS}

Concejo Municipal de Ocamonte, Santander. Acuerdo 009. (29, mayo, 2012). Por el cual se adopta el Plan de Desarrollo del Municipio de Ocamonte, 2012-2015.

Congreso de la República de Colombia. Ley 142 de 1994. Por la cual se establece el régimen de los servicios públicos domiciliarios y se dictan otras disposiciones. Julio 11 de 1994. Diario Oficial $N^{\circ}$. 41.433.

Congreso de la República de Colombia. Ley 388 de 1997. Por la cual se modifican la Ley 9 de 1989 y la Ley 2 de 1991 y se dictan otras disposiciones. Julio 18 de 1997. Diario oficial $\mathrm{N}^{\circ}$. 43.091.

Constitución Política de Colombia. (2012). Bogotá: Leyer.

Corte Constitucional, República de Colombia. Sala Plena. Sentencia C-741 de 2003. Magistrado Ponente: Manuel José Cepeda Espinosa. Expediente D. 4405. Recuperado de: http://www.corteconstitucional.gov.co/ relatoria/2003/c-741-03.htm.

Corte Constitucional, República de Colombia. Sala séptima de revisión. Sentencia de T-413 de 1995. Magistrado ponente: Alejandro Martínez Caballero. Expediente T-71043. Recuperado de: http://www. corteconstitucional.gov.co/relatoria/1995/T-413-95.htm.

Ministerio de Justicia y del Derecho, República de Colombia. Decreto 398 de 2002. Por el cual se reglamenta el inciso $3^{\circ}$ del numeral 6.4 del artículo $6^{\circ}$ de la Ley 142 de 1994. Marzo 4 de 2002. Diario oficial N ${ }^{\circ} .44734$.

Ministerio de Desarrollo Económico, República de Colombia. Decreto 421 de 2000. Por el cual se reglamenta el numeral 4 del artículo 15 de la Ley 142 de 1994, en relación con las organizaciones autorizadas para prestar los servicios públicos de agua potable y saneamiento básico en municipios menores, zonas rurales y áreas urbanas específicas. Marzo 13 de 2000. Diario oficial $\mathrm{N}^{\circ} .43932$. 